\title{
Effectiveness of Muscle Energy Technique Versus Strain Counter Strain Technique on Trigger Points of Quadratus Lumborum among Low Back Pain Patients
}

\author{
SADAF TUBASSAM ${ }^{1}$, SAIMA RIAZ², REHAN RAMZAN KHAN³ ${ }^{3}$ SOBIA GHAFOOR ${ }^{4}$, SAJID RASHID ${ }^{5}$, ANFAL ZAHRA $^{6}$ \\ ${ }^{1}$ Senior Physiotherapist, Sialkot Medical Complex, Sialkot, Pakistan. \\ ${ }^{2}$ Assistant Professor, Riphah College of Rehabilitation and Allied Health Sciences, Riphah International University, Lahore, Pakistan. \\ ${ }^{3}$ Assistant Professor, Multan College of Physiotherapy, Multan Medical \& Dental College, Multan, Pakistan. \\ ${ }^{4}$ Senior Physiotherapist, Ittefaq Hospital Trust, Lahore, Pakistan. \\ ${ }^{5}$ Professor, Multan College of Physiotherapy, Multan Medical \& Dental College, Multan, Pakistan. \\ ${ }^{6}$ Internee Physiotherapist, Riphah College of Rehabilitation and Allied Health Sciences, Riphah International University, Lahore, Pakistan. \\ Correspondence to: Dr. Saima Riaz, MS-OMPT, PhD*, Email: Saima.zahid@riphah.edu.pk, Cell: 0333-4349520
}

\begin{abstract}
Objective: To determine the effectivness of muscle energy technique versus strain counter strain techniques on trigger points of quadratus lumborum among patients with low back pain.

Methodology: A quasi-experimental trial was conducted on 40 patients who had low back pain due to trigger points in Sialkot medical complex hospital, Sialkot. The participants were divided into group A (MET) and group B (SCS). Group A was treated with muscle energy techniques and moist heat therapy, while Group B was treated with strain counter strain technique and moist heat therapy for two weeks. Numeric pain rating scale and Modified Oswestry Disability Questionnaire were used. Data was analyzed on SPSS 21.

Results: The mean score of the Oswestry Disability Index (ODI) was $53.10 \pm 7.35$ and $56.60 \pm 7.89$ at the start of treatment in group A (MET) and group B (SCS), respectively. The final ODI score for group A (MET) and group B (SCS) was $22.25 \pm 5.87$ and $37.70 \pm 7.87$, respectively. The mean value of pain intensity on the Numerical Pain Rating Scale (NPRS) was $7.30 \pm 1.25$ vs. $7.85 \pm 1.26$ at the start of treatment in Group A (MET) and Group B (SCS), respectively. The post-treatment NPRS score was $3.20 \pm 1.16$ vs. $4.55 \pm 1.20$ for Group A (MET) and Group B (SCS), respectively.

Conclusion: According to the findings of this study, both METS and SCS significantly improved pain and functional disability in patients with low back pain caused by trigger points in the quadratus lumborum. However, in terms of mean difference, METS are more effective than SCS.

Keywords: Strain counter strain, METS, Trigger points, Quadratus lumborum, Low back pain
\end{abstract}

\section{INTRODUCTION}

Low Back Pain (LBP) is defined as tiredness, discomfort or pain in the lower back region, radiating to lower extremities unilaterally or bilaterally. (1) Low back pain is a bit common issue that most of the population experience sooner or later in their lives. (2) It is one of the most significant contributors towards disability and causes substantial personal, community and financial burden globally. (3, 4) Low back pain is typically considered to have an 80 percent lifetime prevalence, and it affects from 4-33 percent of the population at any given moment. (5) Myofascial trigger points (MTrP) with a positive jump indication are often regarded as a common cause of LBP. (6) MTrP is a hyperirritable area associated with a stiff band of skeletal muscles, and any pressure or tension to the muscle might result in local or referred pain. (7) Quadratus Lumborum (QL) is a major cause of LBP difficulties, which frequently results in active and latent MTrP. (8) The QL muscle is important in body mechanics and lumbar spine stabilisation.. Poor body mechanics and posture affects body positioning and stresses quadratus lumborum muscle which ultimately leads to myofascial trigger points in muscle. (9)

Manual therapy is a common treatment for severe low back pain. The muscle energy technique is an active technique requiring the participant to contribute in an accurately regulated manner to the voluntary contraction of the muscle of a subject against the counterforce of an operator . (10) Depending on the comparative acuteness of the scenario, the contraction is generally started from or short of a previously identified obstacle of resistance. (11)The mechanisms by which MET generates enhanced ROM is still under study. MET's effectiveness in relaxing the impacted muscles is due to the Golgi tendon organs inhibiting motor activity. Thus the shortened tissues attain a greater degree of ease and extra movement. $(12,13) \mathrm{A}$ growing body of proof indicates that the method of Strain Counterstrain (SCS) is an efficient therapy for MTrP. (14) However, literature on the efficacy of SCS on MTrP in QL in patients with LBP is scarce. The SCS method is an indirect manipulative method for treating muscle trigger points as its therapy action moves away from the restrictive obstacles, i.e. towards ease. (15) It is achieved by putting the tissues in an optimal position of comfort (POC). POC's aim is to decrease tender point irritability and normalize the dysfunction related to tissue. It is established by the soft and atraumatic nature of SCS methods as a secure and efficient primary mode of action in the therapy of painful muscles and the joints that affect them. (16) The intervention to treat chronic low back pain using SCS method can be regarded as a type of manipulative spinal soft tissue therapy because the pelvis, sacrum, and lower limbs are used passively in degrees of flexion, expansion, lateral flexion, and rotation in the placement of the lumbar spine and sacral areas. (17)

Despite the number of treatment modalities detailed in the physiotherapy literature, there is no single conclusive 
treatment that has demonstrated to be effective for treating low back pain originating from trigger points of quadratus lumborum muscle. To overcome this issue, this study was conducted to compare the effectiveness of muscle energy and strain counter strain techniques on myofascial trigger points of quadratus lumborum muscle.

\section{METHODOLOGY}

A Quasi-experimental trial was conducted to compare the effectiveness of Muscle energy technique and strain counter strain technique on pain alleviation and disability modification among patients with painful quadratus lumborum muscle. A total sample of 40 patients, including both males and females aged between 25-40 with localized pain and tenderness at attachments of quadratus lumborum due to the presence of trigger points, were included. The patients with red flag conditions and disc prolapse, spinal stenosis, lumbar radiculopathy and spondylosis were excluded. The participants were randomized into Group A (MET) and Group B (SCS) by using the lottery method. The group A participants were treated with muscle energy techniques, while group $B$ participants were treated with strain counter strain technique. Moist heat therapy was given to both groups as an adjunct. The participants were assessed twice; at pretreatment (week 0 ) and at the termination of treatment (week 2). The total treatment sessions comprise of 10 with the frequency of 5 sessions per week. The duration of each session was 40 minutes with moist heat therapy for 20 minutes and the rest of the time for manual therapy session. After taking ethical approval from the institutional review board, the study was conducted at Sialkot Medical Complex, Sialkot. The data was collected by using standardized tools, including a modified Oswestry disability index and numeric pain rating scale after taking informed consent from the participants. Data was analyzed by using SPSS version 21. Frequency tables and percentages were used for descriptive statistics, while paired and independent samples t-tests were used to compare within and across group differences, respectively. The level of significance ( $p$-value) was set at 0.05 .

\section{RESULTS}

The descriptive characteristics of participants of both groups is shown in table-l. The mean age of patients in

group $A(M E T)$ was $34.35 \pm 4.59$ vs. $34.55 \pm 4.77$ in group $B$ (SCS). The Mean BMl of patients in group A (MET) was $33.57 \pm 4.97$, while in group B (SCS), it was 35.8153 \pm 4.13 . The frequency of females and males in group $A$ (MET) was $12(60 \%)$ and $8(40 \%)$ respectively, while in group B (SCS) frequency of females was $11(55 \%)$ and of the male was 9 (45\%). The frequency of single and married participants in group A (MET) was in 5 (25\%) and 15 (75\%), respectively. While in group B (SCS) frequency of single participants was $4(20 \%)$ and of married participants was $16(80 \%)$ respectively.

Mean ODI and NPRS score of both groups is shown in Table-II. The mean score of the Oswestry Disability Index (ODI) was $53.10 \pm 7.35$ vs. $56.60 \pm 7.89$ at the start of treatment in group A (MET) and group B (SCS), respectively. The final ODI score for group $A(M E T)$ and group B (SCS) was $22.25 \pm 5.87$ vs. $37.70 \pm 7.87$, respectively. The mean value of pain intensity on the Numerical Pain Rating Scale (NPRS) was $7.30 \pm 1.25$ vs. $7.85 \pm 1.26$ at the start of treatment in Group A (MET) and Group B (SCS), respectively. The post-treatment NPRS score was $3.20 \pm 1.16$ vs. $4.55 \pm 1.20$ for Group A (MET) and Group B (SCS), respectively. The p-value on both outcome measures (ODI and NPRS) was <.05 for both groups, showing that both MET and SCS effectively treat patients with low back pain having trigger points on quadratus lumborum muscle. Comparing the mean difference in pre and post-interventional scores of ODI and NPRS, a greater reduction occurred in Group A (MET), so muscle energy technique is more effective than strain counter strain technique in alleviating low back pain originating from trigger points of quadratus lumborum muscle.

Table I: Descriptive Characteristics of Both Groups

\begin{tabular}{|l|l|l|}
\hline & Group A (MET) & Group B (SCS) \\
\hline Age $($ Years $)$ & $34.35 \pm 4.59$ & $34.55 \pm 4.77$ \\
\hline BMI $\left(\mathrm{Kg} / \mathrm{m}^{2}\right)$ & $33.57 \pm 4.97$ & $35.81 \pm 4.13$ \\
\hline Gender & & \\
\hline Male & $8(40.0 \%)$ & $9(45.0 \%)$ \\
\hline Female & $12(60.0 \%)$ & $11(55.0 \%)$ \\
\hline Marital Status & & \\
\hline Single & $5(25.0 \%)$ & $4(20.0 \%)$ \\
\hline Married & $15(75.0 \%)$ & $16(80.0 \%)$ \\
\hline
\end{tabular}

Table II: Mean ODI and NPRS Score across Both Groups

\begin{tabular}{|c|c|c|c|c|c|c|c|c|}
\hline \multirow{2}{*}{$\begin{array}{l}\text { Mean } \\
\text { Scores }\end{array}$} & \multicolumn{4}{|l|}{ Mean ODI Score } & \multicolumn{4}{|c|}{ Mean NPRS Score } \\
\hline & Group A (MET) & $p$-value & Group B (SCS) & p-value & Group A (MET) & p-value & Group B (SCS) & $\mathrm{p}$-value \\
\hline $\begin{array}{l}\text { Baseline } \\
\text { score }\end{array}$ & $53.10 \pm 7.35$ & \multirow{3}{*}{$<0.05$} & $56.60 \pm 7.89$ & \multirow{3}{*}{$<0.05$} & $7.30 \pm 1.25$ & \multirow{3}{*}{$<0.05$} & $7.85 \pm 1.28$ & \multirow{3}{*}{$<0.05$} \\
\hline Final score & $22.25 \pm 5.87$ & & $37.70 \pm 7.87$ & & $3.20 \pm 1.16$ & & $4.55 \pm 1.20$ & \\
\hline $\begin{array}{l}\text { Mean } \\
\text { Difference }\end{array}$ & 30.85 & & 18.90 & & 4.10 & & 3.30 & \\
\hline
\end{tabular}

\section{DISCUSSION}

The current study compared the effects of muscular energy technique vs strain counter strain on quadratus lumborum trigger points in low back pain patients. METS and SCS both showed significant benefits in decreasing pain and functional disability in patients with low back pain caused by quadratus lumborum trigger points. However, in terms of mean difference, METS showed to be more successful than SCS.

Day and Nitz et al. (18) did a study on individuals with low back pain who had a myofascial trigger points in the quadratus lumborum muscle. There were 42 patients in all, divided into two groups. Group A was given the METS technique and moist heat, while Group B was given the 
SCS technique and moist heat. The statistical examination of the groups using the paired-samples t-test revealed significant differences ( $p$ value $<0.05$ ). When the mean differences of the two groups were compared, group $A$ (MET) had a clinically significant difference of 4.10 with a 95 percent confidence interval (3.51387, 4.68613), but group B (SCS) had a mean difference that was not clinically significant.

The current study showed that significant difference exists between both interventions in the treatment of disability and pain. Group A, treated with MET showed more statistically significant results. It aimed to determine the effect of muscle energy versus strain counter strain technique on trigger points of quadratus lumborum in patients with pain. In previous studies, Hariharasudhan R. concluded that Hot moist pack with MET was effective in alleviating mechanical low back pain, increases in lumbar ROM, and reduces disability compared to SCS. (19)

In 2004, Wong CK and Schauer C were surveyed to examine the reliability and validity of the impact of a tenderpoint palpation scale (TPPS) and Strain Counterstrain (SCS) on painful tender-points (TP). All organizations showed important pain reductions with the VAS and TTPS in both muscle groups by the end of the research. The SCS organizations tended to reduce pain. However low reliability and validity of TPPS prevents any data based on this evaluation technique. (14)

Greenman described the muscle energy technique as a controlled manual therapy procedure at different intensity levels relative to the operator's distinctly performed counter force. The objective is to boost joint mobilization and extend contracted muscles. Since no thrusting is performed, this method has a very low possibility of complications and can be used where high velocity is contraindicated with low amplitude. (20)

The current study showed that significant difference exists between both interventions in treatment of disability and pain. Group A, treated with MET showed more statistically significant results. It was aimed to determineeffect of muscle energy technique versus strain counter strain technique on trigger points of quadratus lumborum in patients with pain and the results of this study are coinciding with previous studies.

\section{CONCLUSION}

According to the findings of this study, both METS and SCS significantly improved pain and functional disability in patients with low back pain caused by trigger points in the quadratus lumborum. However, in terms of mean difference, METS are more effective than SCS.

\section{REFERENCES}

1. Golmohammadi K. The cost of low-back pain: a review of the literature. 2003.
2. Itoh $\mathrm{K}$, Katsumi $\mathrm{Y}$, Kitakoji $\mathrm{H}$. Trigger point acupuncture treatment of chronic low back pain in elderly patients-a blinded RCT. Acupuncture in Medicine. 2004;22(4):170-7.

3. Dionne CE, Dunn KM, Croft PR. Does back pain prevalence really decrease with increasing age? A systematic review. Age and ageing. 2006;35(3):229-34 .

4. Struckmann V. Multimorbidität-eine Herausforderung für europäische Gesundheitssysteme. 2019.

5. Dankaerts W, O'sullivan P, Straker L, Burnett A, Skouen J. The inter-examiner reliability of a classification method for non-specific chronic low back pain patients with motor control impairment. Manual therapy. 2006;11(1):28-39.

6. Alvarez DJ, Rockwell PG. Trigger points: diagnosis and management. American family physician. 2002;65(4):653.

7. Simons DG, Travell J, Simons L. Myofascial pain and dysfunction: the trigger point manual. Volume 1. Upper half of body. London, United Kingdom: Williams and Wilkins. 1999;21:22.

8. Huguenin LK. Myofascial trigger points: the current evidence. Physical therapy in sport. 2004;5(1):2-12.

9. McGill SM, Childs A, Liebenson C. Endurance times for low back stabilization exercises: clinical targets for testing and training from a normal database. Archives of physical medicine and rehabilitation. 1999;80(8):941-4.

10. Fryer G, Ruszkowski W. The influence of contraction duration in muscle energy technique applied to the atlantoaxial joint. Journal of osteopathic medicine. 2004;7(2):79-84.

11. Patil PN, Chandu B, Metgud S, Khatri S. Effectiveness of muscle energy technique on quadratus lumborum in acute low back pain-randomized controlled trial. Indian Journal of Physiotherapy and Occupational Therapy. 2010;4(1):54-8.

12. Ballantyne F, Fryer G, McLaughlin P. The effect of muscle energy technique on hamstring extensibility: the mechanism of altered flexibility. Journal of osteopathic medicine. 2003;6(2):59-63.

13. Hamilton L, Boswell C, Fryer G. The effects of high-velocity, low-amplitude manipulation and muscle energy technique on suboccipital tenderness. International journal of osteopathic medicine. 2007;10(2-3):42-9.

14. Wong CK, Schauer C. Reliability, validity and effectiveness of strain counterstrain techniques. Journal of Manual \& Manipulative Therapy. 2004;12(2):107-12.

15. Ibáñez-García J, Alburquerque-Sendín $F$, Rodríguez-Blanco C, Girao D, Atienza-Meseguer A, Planella-Abella S, et al. Changes in masseter muscle trigger points following straincounterstrain or neuro-muscular technique. Journal of bodywork and movement therapies. 2009;13(1):2-10.

16. Naik Prashant $\mathrm{P}$, Anand $\mathrm{H}$, Khatri Subhash M. Comparison of muscle energy technique and positional release therapy in acute low back pain-RCT. Physiotherapy and Occupational Therapy. 2010:32.

17. Wong CK. Strain counterstrain: current concepts and clinical evidence. Manual therapy. 2012;17(1):2-8.

18. Day JM, Nitz AJ. The effect of muscle energy techniques on disability and pain scores in individuals with low back pain. Journal of Sport Rehabilitation. 2012;21(2):194-8.

19. Hariharasudhan R, Balamurugan J. A randomized doubleblinded study of effectiveness of strain counter-strain technique and muscle energy technique in reducing pain and disability in subjects with mechanical low back pain. Saudi Journal of Sports Medicine. 2014;14(2):83.

20. Greenman P. Principles of manual therapy. Baltimore, MD: Williams \& Wilkins; 1996. 